\title{
REVIEW ARTICLE Maternal hyperoxygenation for the human fetus: should studies be curtailed?
}

\author{
Abraham M. Rudolph ${ }^{1}$
}

\begin{abstract}
Congenital hypoplasia of left heart structures in fetuses frequently progresses with gestational development. Interference with cerebral development is common in these fetuses. Chronic maternal hyperoxygenation (MHO) has been recommended to increase left ventricular size and to limit cerebral damage. The effects of $\mathrm{MHO}$ on cerebral blood flow and metabolism have been studied in normal fetuses and fetuses with left heart hypoplasia. Maternal hyperoxygenation increases fetal pulmonary blood flow. This is associated with reduction of foramen ovale flow, thus limiting the increase in left ventricular output. Modest increase in the size of left heart structures has been reported, but in another study, no significant improvement occurred. In sheep fetuses increased oxygenation results in marked reduction of cerebral blood flow, with no change in oxygen delivery or consumption by the brain, but significant reduction in cerebral glucose delivery and consumption. In one study of fetuses with left heart hypoplasia, chronic $\mathrm{MHO}$ was associated with decrease in head size. The effectiveness of MHO in improving left ventricular development is controversial. $\mathrm{MHO}$ is, however, associated with reduction of cerebral blood flow and possible interference with cerebral development. In view of this it is recommended that all studies of chronic maternal hyperoxygenation be curtailed.
\end{abstract}

Pediatric Research (2020) 87:630-633; https://doi.org/10.1038/s41390-019-0604-4

\section{INTRODUCTION}

Oxygen concentrations in fetal blood are considerably lower than those in the mother; they are affected by maternal arterial oxygen concentrations. Umbilical venous blood had a mean $\mathrm{PO}_{2}$ of about $28 \mathrm{mmHg}$ when the mothers were ventilated with air; it increased to about $40 \mathrm{mmHg}$ with ventilation with $100 \%$ oxygen. ${ }^{1}$ The vasculature in many organs of the fetus is sensitive to changes in oxygenation of the blood perfusing the organ. Thus in fetal sheep, decreasing the oxygen tension of arterial blood from 21 to 12 $\mathrm{mmHg}$ by reducing the level of oxygen in maternal inspired air induces an increase in pulmonary vascular resistance with a fall in pulmonary blood flow, a decrease in cerebral vascular resistance with an increase in cerebral blood flow, and a decrease in coronary vascular resistance with an increase in coronary blood flow. ${ }^{2}$ The ability to increase fetal oxygen by providing high oxygen gas mixtures for ventilation by mothers has been considered as a potential treatment for several fetal disorders. Thus it is being recommended as a means of increasing development of the left ventricle and aorta in fetuses with hypoplasia of the left heart or narrow aortic isthmus. Also, because fetal cerebral development has been thought to be impaired by hypoxia, ${ }^{3}$ chronic increase in maternal oxygen levels has been considered as an approach to attempt to limit brain damage.

\section{CHRONIC MATERNAL HYOEROXIA TO IMPROVE LEFT VENTRICULAR AND AORTIC ARCH DEVELOPMENT IN THE FETUS}

Reduction of the volume of blood entering or being ejected by the left ventricle is associated with a decrease in the size of the chamber in fetal lambs. ${ }^{4}$ Progressive hypoplasia of the left ventricle has been reported in human fetuses with aortic stenosis. ${ }^{5}$ The possibility that enhancing venous return to the left ventricle and aorta of the fetus could improve development was proposed by Kohl. ${ }^{6}$ Blood flowing into the left ventricle is derived from pulmonary venous return and flow into the left atrium across the foramen ovale. Kohl suggested that increasing the oxygen concentration in gas inspired by the mother would improve oxygenation of blood in the fetus, induce pulmonary vasodilatation, and thus increase pulmonary venous return and enhance left ventricular output. Fetal pulmonary vasculature is very sensitive to changes in oxygenation; the response is dependent on gestational age. In fetal sheep less than 100 days ( 0.6 gestation), hypoxemia has little effect on pulmonary vascular resistance, but there is then a progressive increase in vascular response to term. ${ }^{7}$ Increasing the oxygen content of fetal blood by placing maternal sheep in a hyperbaric oxygen chamber resulted in a marked increase in fetal pulmonary blood flow. ${ }^{8}$ Studies have also been conducted in human fetuses, by measuring blood flows by Doppler ultrasound techniques. Gestational differences in the response of the pulmonary circulation were also noted. When mothers breathed $60 \%$ oxygen gas mixtures, no change in pulmonary blood flow was noted in fetuses of 20-26 weeks gestation, but in fetuses of 31-36 weeks gestation, pulmonary blood flow increased by a mean of almost $25 \%{ }^{9}$

Although maternal hyperoxygenation (MHO) increases fetal pulmonary blood flow, the effect on total flow to the left ventricle is questionable. If flow through the foramen ovale was maintained, left ventricular filling and output would be increased. However, it is very likely that an increase in pulmonary venous return would be associated with a decrease in flow through the foramen ovale to the left atrium. The pressure gradient across the

${ }^{1}$ Department of Pediatrics, University of California San Francisco, San Francisco, CA, USA

Correspondence: Abraham M. Rudolph (rudolpha@sbcglobal.net)

Received: 3 December 2018 Revised: 25 March 2019 Accepted: 27 June 2019

Published online: 10 October 2019 
pulmonary vasculature is quite large, with a mean pressure in the pulmonary artery of about $45-50 \mathrm{mmHg}$ and in the left atrium of 2-3 mmHg; a small increase in left atrial pressure would thus not greatly affect pulmonary blood flow. However, the pressure gradient between the right and left atrium across the foramen ovale is only $2-3 \mathrm{mmHg}$. Thus, if left atrial pressure is increased only slightly by increased pulmonary venous return, flow across the foramen ovale would be significantly reduced. Thus MHO may somewhat increase left ventricular output, but the volume would be lower than what might be expected from the increase in pulmonary blood flow. In studies on human fetuses done by phase-contrast MRI, there was an inverse relationship between pulmonary blood flow and foramen ovale shunt. ${ }^{10}$ Also in the study by Rasanen et al., MHO increased pulmonary blood flow in the older fetuses, but foramen ovale flow decreased and left ventricular output was not altered significantly. ${ }^{9}$ Similarly a fetus with left ventricular hypoplasia, studied by phase-contrast MRI, developed an increase in pulmonary blood flow with $\mathrm{MHO}$, but left ventricular output did not improve. ${ }^{11}$

Several studies to attempt to improve the development of left-sided cardiac structures by $\mathrm{MHO}$ in fetuses with various congenital cardiovascular malformations have been reported. Kohl observed the effects of hyperoxygenation on 15 fetuses, 13 of which had left ventricular or aortic hypoplasia, during the gestational period of 33-38 weeks. Hyperoxygenation was provided for three 4-h periods per day for 8-33 days, until spontaneous delivery. Left ventricular area increased in seven fetuses and mitral and aortic valve diameters increased in many. Aortic arch diameter showed a striking increase in most of the fetuses. ${ }^{6}$ These results were promising, but could not be confirmed by the study of Lara et al. ${ }^{12}$ Oxygen was administered to mothers of nine fetuses with left heart hypoplasia for at least $8 \mathrm{~h}$ per day, starting at 26-34 weeks gestation and provided for 33-84 days. During oxygen therapy, maternal arterial $\mathrm{Po}_{2}>$ $250 \mathrm{mmHg}$ was achieved. Nine fetuses with left heart hypoplasia served as controls. In the fetuses subjected to MHO, there was a trend toward increase in both mitral and aortic annular dimension. However, none of the measurements showed statistical significance as compared with the control group.

The effects of MHO on fetuses with aortic coarctation have been reported by Zeng et al. ${ }^{13}$ Of 48 fetuses with coarctation, 24 were subjected to treatment and 24 served as controls. After 4 weeks of therapy, Z-scores of aortic valve, mitral valve and aortic arch dimension were statistically greater in the fetuses in the hyperoxemia group. However, beyond 4 weeks, differences between aortic and mitral valve dimensions were no longer significantly different, but aortic arch dimensions were improved.

\section{EFFECT OF MATERNAL HYPEROXEMIA ON THE BRAIN}

Cerebral blood flow is very sensitive to the oxygen content of perfusing blood. In fetal sheep, reducing the carotid arterial $\mathrm{Po}_{s}$ from 21 to $12 \mathrm{mmHg}$ by providing low oxygen gas mixtures for ventilation by the mother resulted in a 2 to 3 -fold increase in cerebral blood flow. ${ }^{2}$ In the adult human, breathing $100 \%$ oxygen results in a $26 \%$ reduction in cerebral blood flow. ${ }^{14}$ Studies by BOLD-MRI, which is considered to reflect tissue oxygenation, showed an increase in levels in the liver, kidneys and placenta, but not in the brain, in human fetuses with gestational ages of 28-34 weeks as a result of $\mathrm{MHO}^{15}$ Pulsatility index (PI), as measured in blood flow recordings by Doppler ultrasound in cerebral arteries, has been used as a means of assessing cerebral vascular resistance. An increase in the $\mathrm{PI}$ is considered to reflect vasoconstriction and a decrease in $\mathrm{PI}$ denotes vasodilation. In a study of human fetuses, $\mathrm{MHO}$ increased cerebral PI; the effect was mild and variable at 20-23 weeks gestation, but marked and consistent at $30-33$ weeks gestation. ${ }^{16}$ During the hyperoxygenation, aortic isthmus PI was decreased. The authors suggested that $\mathrm{MHO}$ "results in an increase in cerebral vascular resistance and redistribution of blood flow from the brain to the vascular beds supplied by the descending aorta".

During fetal life, the brain is perfused by ascending aortic blood with an oxygen saturation of about $65 \%$ in the lamb; after birth the saturation increases to almost $100 \%$. We examined the effect of acute change in oxygen saturation on cerebral blood flow and metabolism. ${ }^{17}$ Fetal sheep were prepared with indwelling vascular catheters and a tracheal tube. After recovery for 2-3 days studies were conducted on the fetuses in utero; blood flows to organs were measured by the radionuclide-labeled microsphere technique. Measurements were made under control conditions, then during ventilation of the fetus, first with $3 \%$ oxygen and then with $100 \%$ oxygen. Mean values for cerebral blood flow, carotid arterial oxygen saturation and glucose concentration, and oxygen and glucose delivery to and consumption by the brain are shown in Table 1. No significant changes were noted during ventilation with $3 \%$ oxygen. Ventilation with $100 \%$ oxygen resulted in a marked increase in carotid arterial oxygen saturation. Cerebral blood flow fell dramatically to $32 \%$ of control values. Because arterial oxygen levels increased, the oxygen delivery to and consumption by the brain fell slightly, but not significantly. Blood glucose concentration did not change significantly with ventilation with oxygen, so the marked reduction in cerebral blood flow resulted in a $56 \%$ fall in glucose delivery to, and $27 \%$ reduction in glucose consumption by, the brain.

The effect of acute $\mathrm{MHO}$ on the brain has been studied in 43 human fetuses with left heart hypoplasia. ${ }^{18}$ Middle cerebral artery

Table 1. Fetal sheep were ventilated in utero, first with $3 \%$ oxygen, which did not change arterial oxygen saturation and then with $100 \%$ oxygen; this markedly increased arterial oxygen saturation

\begin{tabular}{llll}
\hline & Control & Ventilation 3\% oxygen & Ventilation 100\% oxygen \\
\hline Cerebral blood flow, $\mathrm{ml} / \mathrm{min} / 100 \mathrm{~g}$ & 144 & 102 & $46^{*}$ \\
Cerebral arterial oxygen saturation, $\%$ & 40 & 42 & $96^{*}$ \\
Oxygen delivery, $\mathrm{mM} / \mathrm{min} / 100 \mathrm{~g}$ & 402 & 274 & 330 \\
Oxygen consumption, $\mathrm{mM} / \mathrm{min} / 100 \mathrm{~g}$ & 173 & 104 & 145 \\
Glucose content, $\mathrm{mM}$ & 1.1 & 1.4 & 1.4 \\
Glucose delivery, $\mathrm{mM} / \mathrm{min} / 100 \mathrm{~g}$ & 138 & 146 & $61^{*}$ \\
Glucose consumption, $\mathrm{mM} / \mathrm{min} / 100 \mathrm{~g}$ & 20.9 & 22.8 & $15.2^{*}$ \\
\hline $\begin{array}{l}\text { Cerebral blood flow did not change significantly with ventilation with 3\% oxygen, but fell markedly with } 100 \% \text { oxygen ventilation. Oxygen delivery to the } \\
\text { brain and cerebral oxygen consumption did not change significantly during ventilation with either 3 or 100\% oxygen. Cerebral glucose delivery and } \\
\text { consumption both fell markedly with ventilation with } 100 \% \text { oxygen }\end{array}$ & & \\
$\begin{array}{l}\text { The data for this table are derived from ref. }{ }^{*} \\
\text { *Significantly different } p<0.5\end{array}$ & \\
\hline
\end{tabular}


pulsatility index (PI) was lower than normal in these fetuses and the extent of this reduction increased with gestational age. This suggested that cerebral vascular resistance was low. With hyperoxygenation the PI Z-scores increased, particularly in fetuses above 28 weeks gestation. The increase in PI was also more prominent, the lower the baseline PI. The authors suggest that the fetuses with the lowest PI had maximal vasodilation. Providing oxygen increased arterial oxygen content and thus oxygen delivery could be maintained with a lower cerebral blood flow. It was proposed that the increase in cerebral vascular resistance associated with MNO was a beneficial response that "enhanced neuroprotection".

The effect of chronic $\mathrm{MHO}$ on cerebral development was studied in nine fetuses with left heart hypoplasia, with nine fetuses with hypoplasia serving as controls. ${ }^{19}$ In the fetuses subjected to hyperoxia, skull biparietal diameter growth was significantly slower than in control fetuses. Also, postnatally, at 6 months, head circumference was significantly smaller in these fetuses. Neuro-developmental testing at 6 and 12 months after birth showed no differences between the two groups.

\section{DISCUSSION}

The term maternal hyperoxygenation (MHO) has been applied to the practice of providing mothers with inhaled gas mixtures with increased oxygen concentrations. This has increased maternal arterial $\mathrm{Po}_{2}$ levels from about $100 \mathrm{mmHg}$ to about $250 \mathrm{mmHg}$, also resulting in an increase in arterial oxygen content of fetal blood. As mentioned above, numerous studies on animal and human fetuses have demonstrated the marked effects of changes in oxygen content on vascular resistance in several organs. The possibility that these changes could be applied to treat several fetal anomalies has led to initiation of studies on human fetuses. Currently, the major interests relate to the response of the pulmonary circulation and to the oxygen content of cerebral arterial blood.

Maternal hyperoxygenation has been shown to increase pulmonary blood flow in human fetuses; this response is dependent on gestational age, being noted beyond about 25 weeks gestation. It has been proposed that an increase in pulmonary flow may be useful in improving left ventricular development in fetuses with left-sided diaphragmatic hernia. ${ }^{20}$ The main interest has been directed to the possibility that chronic MHO could improve development of left cardiac chambers and the aorta in fetuses with hypoplasia of these structures. Since the left ventricle receives blood from the lungs and the foramen ovale, the effectiveness of greater pulmonary venous return in increasing left ventricular filling and output would be dependent on the relationship between pulmonary blood flow and foramen ovale flow. Studies in human fetuses showed that $\mathrm{MHO}$ resulted in a reduction of foramen ovale flow into the left heart that was proportional to the increase in pulmonary flow, so that left ventricular output was not altered. ${ }^{9}$ Despite this observation, studies by Kohl on chronic hyperoxygenation of human fetuses with left cardiac hypoplasia showed improvement of left ventricular size and of mitral and aortic valve areas. ${ }^{6}$ However, in comparing the response to MHO of a group of fetuses with left heart hypoplasia to a group with hypoplasia that was not treated, there were no significant differences. $^{13}$ These authors, in a response to a letter to the journal, stated "given our results, we cannot claim that $\mathrm{CMH}$ in its current form should be used clinically, as the effect sizes in this pilot study were small".

Neurobehavioral abnormalities are prevalent in some newborn infants with congenital cardiovascular malformations, ${ }^{21}$ suggesting that cerebral damage has occurred prior to birth. Sun et al. proposed that impaired brain growth in fetuses with congenital cardiac disorders was related to reduced cerebral oxygenation and raised the possibility that cerebral development could be improved by maternal oxygen therapy. ${ }^{22}$ In the study by Szwast et al., ${ }^{16}$ fetuses with left heart hypoplasia were noted to have low cerebral arterial pulsatility indices $(\mathrm{PI})$, which increased during MHO. They proposed that this was due to an increase in fetal cerebral arterial oxygen content, which resulted in cerebral vasoconstriction.

They suggested that the increase of PI to more normal values was a positive response and that this "enhanced neuroprotection". This is based on the concept that cerebral damage is related to inadequate supply of oxygen to the brain. However, as mentioned above, based on the studies in fetal lambs, increasing oxygen concentration in cerebral arterial blood results in vasoconstriction with reduction in cerebral blood flow. ${ }^{17}$ Although cerebral oxygen delivery and consumption are maintained, glucose delivery and consumption are markedly reduced. The reduction in substrate could interfere with cerebral development, despite an adequate oxygen supply. ${ }^{23}$

The possibility that MHO may indeed have an adverse effect on cerebral development is supported by the report of Edwards et al., ${ }^{19}$ showing slow growth of skull biparietal diameter during fetal life and reduced head circumference postnatally in fetuses with hypoplastic left heart subjected to $\mathrm{MHO}$, compared with fetuses with hypoplastic left heart that served as controls. Considering the fact that a beneficial effect of chronic MHO on left ventricular development is questionable and that it possibly interferes with cerebral development, I propose that studies of the use of MHO administered repeatedly for several hours a day be curtailed.

\section{ADDITIONAL INFORMATION}

Competing interests: The author declares no competing interests.

Publisher's note Springer Nature remains neutral with regard to jurisdictional claims in published maps and institutional affiliations.

\section{REFERENCES}

1. Ramanathan, S., Gandhi, S., Arismendy, J., Chalon, J. \& Turndorf, H. Oxygen transfer from mother to fetus during epidural anesthesia. Anesth. Analg. 61, 576-581 (1982).

2. Cohn, H. E., Sacks, E. J., Heymann, M. A. \& Rudolph, A. M. Cardiovascular responses to hypoxemia and acidemia in fetal lambs. Am. J. Obstet. Gynecol. 120, 817-824 (1974).

3. Bsburamani, A. A., Ek, C. J., Walker, D. W. \& Castillo-Melendez, M. Vulnerability of the developing brain to hypoxic-ischemic damage: contribution of the cerebral vasculature to injury and repair?. Front. Physiol. 3, 424 (2012).

4. Fishman, N. H., Hof, R. B., Rudolph, A. M. \& Heymann, M. A. Models of congenital heart disease in fetal lambs. Circulation 58, 354-364 (1975).

5. Yamamoto, Y. \& Hornberger, L. K. Progression of outflow tract obstruction in the fetus. Early Hum. Dev. 88, 279-285 (2012).

6. Kohl, T. Chronic intermittent materno-fetal hyperoxygenation in late gestation may improve on hypoplastic vascular structures associated with cardiac malformations in human fetuses. Pediatr. Cardiol. 31, 250-263 (2010).

7. Lewis, A. B., Heymann, M. A. \& Rudolph, A. M. Gestational changes in pulmonary vascular responses in fetal lambs in utero. Circ. Res. 39, 536-541 (1976).

8. Heymann, M. A., Rudolph, A. M. \& Melmon, K. L. Bradykinin production associated with oxygenation of the fetal lamb. Circ. Res. 25, 521-534 (1969).

9. Rasanen, J. et al. Reactivity of the human fetal pulmonarycirculation to maternal hyperoxygenation increases during the second half of pregnancy: a randomized study. Circulation 97, 257-262 (1998).

10. Prsa, M. et al. Reference ranges of blood flow in the major vessels of the normal human fetal circulation at term by phase-contrast magnetic resonance imaging. Circ. Cardiovasc. Imaging 7, 663-670 (2014).

11. Borik, S., Macgowan, C. K. \& Seed, M. Maternal hyperoxygenation and foetal cardiac MRI in the assessment of the borderline left ventricle. Cardiol. Young $\mathbf{2 5}$, 1214-1217 (2015).

12. Lara, D. A. et al. Pilot study of chronic maternal hyperoxygenation and effect on aortic and mitral valve annular dimensions in fetuses with left heart hypoplasia. Ultrasound Obstet. Gynecol. 48, 365-372 (2016). 
Maternal hyperoxygenation for the human fetus: should studies be curtailed? AM. Rudolph

13. Zeng, S. et al. Sustained maternal hyperoxygenation improves aortic arch dimensions in fetuses with coarctation. Sci. Rep. 16, 39304 (2016).

14. Watson, N. A., Beards, S. C., Allaf, N., Kassner, A. \& Jackson, A. The effect of hyperoxia on cerebral blood flow: a study in healthy volunteers using magnetic resonance phase-contrast angiography. Eur. J. Anaesthesiol. 17, 132-139 (2000).

15. Sorensen, A. et al. Changes in fetal oxygenation during maternal hyperoxia as estimated by BOLD MRI. Prenat. Diagn. 33, 141-145 (2013).

16. Almstrom, H. \& Sonesson, S. E. Doppler echo-cardiographic assessment of fetal blood flow distribution during maternal hyperoxygenation. Ultrasound Obstet. Gynecol. 8, 256-261 (1996).

17. Iwamoto, H. S., Teitel, D. F. \& Rudolph, A. M. Effect of birth-related events on metabolism in fetal sheep. Pediatr. Res. 30, 158-164 (1991).

18. Szwast, A., Putt, M., Gaynor, J. W., Licht, D. J. \& Rychik, J. Cerebrovascular response to maternal hyperoxygenation in fetuses with hypoplastic left heart syndrome depends on gestational age and baseline cerebrovascular resistance. Ultrasound Obstet. Gynecol. 52, 473-478 (2018).

19. Edwards, L. A. et al. Chronic maternal hyperoxygenation and effect on cerebral and placental vasoregulation and neurodevelopment in fetuses with left heart hypoplasia. Fetal Diagn. Ther. 46, 45-57 (2019).

20. Ishii, R. et al. Effects of maternal hyoeroxygenation in a case of severe congenital diaphragmatic hernia accompanied by hydrops fetalis. J. Ped Surg. 2, 15-19 (2014).

21. Limperopoulos, C. et al. Neurologic status of newborns with congenital heart defects before open heart surgery. Pediatrics 103, 402-408 (1999).

22. Sun, L. et al. Reduced fetal cerebral oxygen consumption is associated with smaller brain size in fetuses with congenital heart disease. Circulation 131, 1313-1323 (2015).

23. Rudolph, A. M. Impaired cerebral development in fetuses with congenital cardiovascular malformations: is it the result of inadequate glucose supply? Pediatr. Res. 80, 172-177 (2016) 